Service social

\title{
Liens et chaînons manquants dans le processus d'utilisation de la recherche. Utilisation accrue grâce aux méthodes de recherche et développement (Traduction de René Auclair)
}

\author{
Jack Rothman
}

Volume 47, numéro 1-2, 1998

Évaluation - Colloque 1999

URI : https://id.erudit.org/iderudit/706790ar

DOI : https://doi.org/10.7202/706790ar

Aller au sommaire du numéro

Éditeur(s)

École de service social de l'Université Laval

ISSN

1708-1734 (numérique)

Découvrir la revue

Citer cet article

Rothman, J. (1998). Liens et chaînons manquants dans le processus d'utilisation de la recherche. Utilisation accrue grâce aux méthodes de recherche et développement (Traduction de René Auclair). Service social, 47(1-2), 235-276. https://doi.org/10.7202/706790ar
Résumé de l'article

Après une introduction de base portant sur la R-D sociale, Jack Rothman propose dans son article un modèle de recherche et développement des services d'aide aux personnes. Pour ce faire, il présente d'abord le contexte et l'environnement de la R-D. À l'aide d'une recherche documentaire il se réfère ensuite aux connaissances en sciences sociales, puis formule les concepts dans une perspective de recherche appliquée. Il conçoit les études pilotes et conduit les études sur le terrain. Il produit la R-D proprement dite; il la diffuse et la met en marché, tout en créant les structures et les ressources nécessaires aux centres de R-D sociale. 


\section{Évaluation, recherche et développement}

\section{Liens et chaînons manquants dans le processus d'utilisation de la recherche Utilisation accrue grâce aux méthodes de recherche et développement}

Modèle de R-D sociale (Jack Rothman)

Après une introduction de base portant sur la R-D sociale, Jack Rothman propose dans son article un modèle de recherche et développement des services d'aide aux personnes. Pour ce faire, il présente d'abord le contexte et l'environnement de la R-D. À l'aide d'une recherche documentaire il se réfère ensuite aux connaissances en sciences sociales, puis formule les concepts dans une perspective de recherche appliquée. Il conçoit les études pilotes et conduit les études sur le terrain. II produit la R-D proprement dite; il la diffuse et la met en marché, tout en créant les structures et les ressources nécessaires aux centres de R-D sociale.

Following a basic introduction to social $R-D$, paper is devoted to a research and development model for the human services, the context and environment of $R-D$, retrieval of existing social research knowledge, formulating application concepts, pilot testing, main field testing, the product of social $R-D$, diffusion and social marketing, and structures and resources for centers of social $R-D$.

1. Cet article est tiré d'un recueil de textes inédits sur l'utilisation de la recherche en service social, Allen Rubin et Aaron Rosenblatt (dir.), traduits par René Auclair et Louise Tremblay, Québec, Les Presses de l'Université Laval, 1984, p. 155-209 (épuisé). 


\section{REMARQUE PRÉLIMINAIRE}

Les membres du comité de rédaction jugent pertinent de publier à nouveau un texte de Jack Rothman, paru en français aux Presses de l'Université Laval en 1984 et épuisé, puisque c'est un classique dans le domaine de la recherche et de ses applications relatives aux programmes et à l'intervention où s'insère l'évaluation. En effet, selon ce cadre de référence, l'auteur conçoit la recherche et l'intervention comme un continuum, c'est-à-dire un ensemble d'éléments tels que l'on peut passer de l'un à l'autre de façon continue. Sont abordés dans ce texte à la fois les stratégies et les concepts essentiels du transfert des connaissances en tant que processus interactif, mettant en lien des décideurs, des intervenants et des chercheurs qui peuvent être producteurs de connaissances aussi bien qu'utilisateurs.

Dans le processus de transfert de connaissances, il convient également de bien distinguer les cibles qui seront l'objet d'un tel transfert. Les stratégies seront différentes selon que l'utilisateur potentiel appartient au milieu de la planification et a besoin des résultats de la recherche pour la prise de décision, ou selon que l'utilisateur potentiel appartient au milieu de la pratique pour qui les résultats de la recherche servent à l'intervention clinique.

\section{INTRODUCTION}

Plusieurs questions, auxquelles nous pourrions peut-être répondre, ont été soulevées, notamment :

- Que pourrait-on suggérer pour réduire l'écart de traduction mentionné par P.F. Lazerfeld?

- Comment la recherche en service social est-elle structurée?

- Au sein des organismes nationaux de service social, quels changements faudrait-il apporter pour améliorer l'utilisation de la recherche?

Là ne s'arrêtaient pas les questions; nous avons cependant choisi de les circonscrire, puisqu'il s'agit déjà d'une entreprise prodigieuse pour un article d'une telle nature.

Notre analyse aura l'écart de traduction pour objet fondamental : nous nous proposons d'en traiter de façon très exhaustive afin d'examiner l'écart plus global entre la recherche, d'une part, et ses applications 
au niveau des programmes et de l'intervention, d'autre part. Nous offrirons une solution pour combler cette lacune qui gêne les travailleurs sociaux depuis si longtemps. Pour y parvenir, il faudra considérer le processus d'utilisation de la recherche comme l'enchaînement de diverses étapes, de différents rôles et de fonctions techniques distinctes. Notre schéma fera la démonstration des mécanismes et des règles pouvant servir à rattacher les résultats de la recherche en sciences sociales aux activités d'intervention liées aux services individualisés.

Pour la présentation de ce schéma, nous puiserons certaines données dans l'un de nos ouvrages antérieurs qui s'intitule Planning and Organizing for Social Change $e^{2}$. Au cours des années qui ont suivi sa publication, nous l'avons raffiné et mis à jour, à la lumière de la compréhension et de l'expérience que nous avons acquises - notre personnel également - grâce à nos recherches dans le cadre du Projet d'intervention communautaire ${ }^{3}$, programme de recherche appliquée que nous avons dirigé au cours de la dernière décennie et à l'intérieur duquel nous avons utilisé le modèle.

Du début du présent article jusqu'à la fin, nous ferons souvent référence à certains termes familiers que nous utiliserons dans un contexte peu familier. Le premier d'entre eux, " recherche et développement », est une expression qu'on a tendance à associer plus généralement à la technologie industrielle et aux sciences physiques. Selon nous, cependant, c'est grâce à un schéma de recherche et développement qu'on pourra obtenir le cadre conceptuel optimal pour la définition des éléments et des possibilités inhérents au processus d'utilisation de la recherche dans son application à la solution des problèmes humains. Le second terme que nous utiliserons peut engendrer beaucoup de passions, surtout lorsqu'on le juxtapose d'une façon quelconque à la profession du service social : il s'agit du terme "ingénierie ", plus spécialement de l'expression " ingénierie sociale ». Nous examinerons de façon exhaustive notre conception du rôle du spécialiste en ingénierie sociale subséquemment. Les lecteurs auront alors une idée générale de ses fonctions éventuelles dans le processus d'utilisation de la recherche.

2. Jack Rothman, Planning and Organizing for Social Change : Action Principles from Social Research, New York, Columbia University Press, 1974.

3. Note du traducteur : Community Intervention Project. 
Dans notre article, nous tenterons surtout d'illustrer comment la recherche sociale pourrait servir à détecter les brèches à l'intérieur du processus d'utilisation lui-même; nous essaierons de voir ce qu'il faut faire du point de vue structural et technique pour les colmater. Nous traiterons, pour conclure, des implications d'un pareil modèle de juxtaposition du processus de recherche et développement au processus d'utilisation de la recherche, pour la profession du service social et pour la structuration de la recherche au sein des services d'aide individualisée.

\section{DICHOTOMIE RECHERCHE-INTERVENTION}

En sciences sociales, l'ensemble des connaissances recueillies par les chercheurs au cours des années et virtuellement utiles est vaste et s'accroît avec rapidité. Les concepteurs de programmes de services sociaux et les praticiens y puisent médiocrement et gauchement. La nature même des responsabilités des professionnels engagés dans les services d'aide individualisée est telle que, sur le plan pratique, ils n'ont guère le temps ni l'occasion de fouiller dans le réservoir des connaissances pour y chercher ce qui pourrait leur être utile ni de mettre en application, de façon rigoureuse, ce qui s'y trouve. Ils n'ont pu jusqu'ici avoir accès à une connaissance sous une forme utilisable et ce, promptement, aisément et efficacement.

Le spécialiste de la recherche fondamentale en sciences sociales et le professionnel des services sociaux sont divisés par suite d'un besoin commun : l'utilisation efficace des connaissances en sciences sociales. Une telle controverse n'a pas été sans retombées. Les praticiens attendent impatiemment que les chercheurs leur fournissent certaines données sous forme pratique, et ceux-ci leur présentent en fait des données abstraites par l'intermédiaire de revues, d'ouvrages, de communications scientifiques dans le cadre de congrès et d'études monographiques, etc. Ils se considèrent comme efficaces dans la transmission de leurs résultats; ils s'inquiètent et s'irritent souvent de ce que les praticiens ne tirent pas profit de ce qui est offert. Un climat latent de frictions et de défiance s'insinue alors dans la relation entre chercheurs et praticiens : conséquence de mauvaises perceptions omniprésentes de leurs rôles et de leurs fonctions respectifs.

Pour les scientifiques sociaux, les praticiens n'accordent guère de valeur à l'intellectualité, mais beaucoup à l'action ou au changement 
pour lui-même. Au lieu d'examiner de façon ordonnée et rigoureuse certaines questions qui nécessitent temps et objectivité scientifique, les praticiens donnent l'impression d'en venir à des conclusions hâtives et de poser des gestes qui ne sont pas corroborés par des données suffisantes. Aux yeux du scientifique social, ils ne s'intéressent nullement à l'autocritique et s'offusquent des critiques venant de l'extérieur; ils se méfient et craignent la recherche. Une telle attitude est également reliée à un professionnalisme étroit : ils s'impliquent tellement au niveau des détails d'une situation problématique donnée qu'ils ne peuvent effectuer des généralisations plus abstraites d'un contexte à l'autre. Les scientifiques sociaux s'indignent également de la suffisance des praticiens qui se croient les seuls à comprendre les problèmes matériels ou le monde ordinaire en raison de leur implication directe et quotidienne. Ils soutiennent, en outre, que c'est aux praticiens qu'il incombe de tirer des conclusions utiles sur le plan des grands principes et de l'action, non pas à eux.

Cette perception est manifestement faussée. Toutefois, la perception du praticien lui-même l'est également.

Pour les praticiens, les scientifiques sociaux effectuent des études sans grande applicabilité sur le plan social. Selon eux, les thèmes étudiés sont asociaux, insignifiants, hermétiques ou abstraits, dans une large mesure, et ils reflètent plus un scientisme étroit que des préoccupations humanistes. Si leurs travaux expriment certaines valeurs sociales, ils sont souvent teintés de conservatisme. Suivant le praticien, la théorie systémique dans son ensemble est un exemple probant de ce statisme. Les scientifiques sociaux semblent faire fi des retombées de leurs travaux au niveau des programmes, et leur incapacité à relier les résultats de leurs recherches à des problèmes sociaux importants dépend, croit-on, d'une certaine arrogance ou d'un manque de compassion. Ils font aussi certaines concessions quant à leur intégrité intellectuelle, alors qu'ils subordonnent leurs travaux de recherche à des contraintes momentanées : disponibilité des subventions du gouvernement central ou de fondations quelconques, ou thèmes de recherche qui présenteront le plus d'avantages pour eux sur le plan de l'argent et du prestige professionnel.

Aux yeux du praticien, la concentration des scientifiques sociaux sur des " trucs " méthodologiques et sur le jargon grandiloquent qui les caractérise ne fait que repousser ceux qui veulent sérieusement se 
servir des connaissances en sciences sociales. Dans les ouvrages de cette discipline, ils voient une certaine élasticité au point de vue des conclusions à atteindre. Les praticiens sont intéressés par des réponses précises à des questions particulières, alors qu'ils n'y trouvent que des généralités peu concluantes sur des sujets très étendus.

II est bien évident que chercheurs et praticiens sont divisés par des styles et par des modes de penser différents, ce qui complexifie leur communication et l'utilisation de leurs contributions et de leurs produits respectifs. La distance sociale qui les sépare est énorme, et elle se caractérise par la méfiance, par les divergences de points de vue et par des objectifs visiblement antagonistes. Par le passé, elle a empêché une véritable imbrication des travaux de l'un et l'autre groupe.

Peut-être la distinction fondamentale se situe-t-elle au niveau du rôle. Les scientifiques sociaux ont pour fonction première de saisir le monde, donc de produire des connaissances qui leur permettent, ainsi qu'à d'autres, de le comprendre mieux. Pour leur part, les praticiens ont pour rôle de transformer le monde (ou certaines parties plus particulièrement), c'est-à-dire de créer des effets d'une certaine tangibilité qui permettent à la clientèle, aux organisations ou aux collectivités de fonctionner davantage en accord avec certains résultats souhaités.

\section{RECHERCHE ET DÉVELOPPEMENT : SOLUTION ET CHARNIËRE}

On peut décortiquer les faits en faisant une certaine analogie avec une opération de tronçonnage de bois. Supposons que les chercheurs en sciences sociales se soient rendus dans la forêt de la connaissance, qu'ils y aient abattu plus d'un arbre solide et se soient montré réciproquement les fruits de leurs excellents travaux. Quelques bûcherons hardis et travailleurs ont traîné certaines bûches jusqu'au cours d'eau et les y ont poussées en aval (ce qu'on désigne sous le nom de " diffusion »). Quelque part en aval, les praticiens au service de certaines compagnies de construction parviennent d'une façon quelconque à bâtir quelques bâtiments de fortune avec ce qui a pu y dériver : mais, dans l'ensemble, ils manquent grandement des pièces de bois de charpente, de tailles et de formes diverses, nécessaires pour faire un travail convenable. Le problème : quelqu'un a omis de construire un moulin pour convertir les bûches en bois de charpente sous toutes 
ses formes utilisables; elles continuent donc de s'amonceler à une extrémité de la chaîne, tandis que les compagnies de construction poursuivent l'érection de bâtiments de fortune à l'autre extrémité.

Pour pousser cette analogie plus loin encore, supposons que gouvernements et fondations aient soutenu financièrement l'opération de tronçonnage, de même que les compagnies de construction. Cependant, presque rien n'a été mis en œuvre pour l'aménagement et pour l'exploitation d'un moulin qui correspond, selon nous, à la méthodologie de recherche et développement en sciences sociales. II s'agit fondamentalement d'un processus systématique pour colmater la brèche entre la recherche et ses applications. II permet la conversion de principes scientifiques en instruments de travail et en lignes d'action spécifiques pour pouvoir s'occuper des véritables problèmes du monde. Cela, Harvey Brooks (1965, p. 126) l'énonce simplement sous forme d'un questionnement :

... Comment peut-on utiliser la connaissance compilée dans le seul but de connaître - recherche fondamentale? Comment peut-on condenser, résumer, reconstituer et interpréter cette connaissance pour pouvoir l'utiliser? Comment peut-on la communiquer aux responsables de l'action : décideurs, novateurs sur le plan technologique, professionnels œuvrant au niveau des services, étudiants?

Dans une évaluation succincte, Richard E. Schutz (1979, p. 39) se concentre sur " l'écart qui existe entre les connaissances scientifiques et les habitudes des utilisateurs ». Launor F. Carter (1968, p. 17), pour sa part, souligne fortement la nécessité de créer des maillons manquants :

Dans l'évaluation des problèmes contemporains en pédagogie et dans l'ensemble des disciplines sociales, il semble y avoir une nette dissociation entre les praticiens œuvrant dans ces domaines et les chercheurs universitaires. II n'existe pas d'intermédiaires qui, à l'instar de l'ingénieur, se consacrent à la résolution de problèmes spécifiques... De telles personnes manquant en pédagogie et dans les disciplines sociales.

Un autre spécialiste, Norman J. Boyan (1968, p. 22), fait remarquer que

la plupart des observateurs se sont limités à l'analyse de la variabilité des facteurs en présence. Seuls quelques-uns se sont demandé s'il manquait certains éléments essentiels... dans l'ensemble du 
processus de conversion des connaissances théoriques au niveau de la pratique.

Par leurs travaux, les membres du Projet d'intervention communautaire en R-D sociale s'efforcent précisément de déterminer ces éléments manquants. Aussi convient-il ici d'en faire une brève présentation.

Le Projet d'intervention communautaire est un programme de recherche et développement en cours. Son but premier est de fournir des outils d'intervention axés sur la recherche et éprouvés sur le terrain aux professionnels qui travaillent dans le domaine des services sociaux reliés à la collectivité.

Ses travaux ont consisté en une succession d'étapes liées sur le plan opérationnel. Les trois premières années (1968-1971) ont nécessité un dépouillement exhaustif des ouvrages de sciences sociales et des publications professionnelles, pour y extraire certaines découvertes, axées sur la recherche, applicables sur le plan de l'intervention communautaire et organisationnelle. Ces conclusions ont servi de base à l'élaboration d'une quantité considérable de généralisations et de sousgénéralisations, chacune étant ensuite convertie sous une forme appliquée. II en est donc résulté un ensemble de "principes d'action » ou de stratégies utilisables par les agents de changement professionnels, par les praticiens, par les administrateurs d'agences et par les planificateurs.

La seconde étape (1971-1974) a servi à la mise en œuvre d'un certain nombre de ces principes d'action au moyen d'une expérimentation sur le terrain. Au cours de cette vérification, nous nous sommes efforcés de les rendre opérationnels et de les évaluer dans des cadres d'application réalistes. Nous avons projeté, en outre, la préparation d'un manuel du praticien qui présenterait spécifiquement les étapes, les ressources et les contacts personnels et organisationnels, etc., nécessaires à leur mise en application dans les agences de services sociaux.

À l'étape suivante (1974-1977), nous nous sommes concentrés surtout sur la révision du manuel en l'adaptant tout particulièrement au personnel œuvrant dans le domaine de la santé mentale. Nous avons aussi étudié divers moyens de diffuser les produits de la R-D sociale auprès de la population d'utilisateurs virtuels dans son ensemble. 
L'objectif ultime des membres du projet est d'en arriver à une implantation solide des méthodes de R-D issues de l'expérience du projet lui-même, par la création d'un laboratoire de R-D permanent, imbriqué dans un réseau national de distribution de senvices individuels. Les problèmes du réseau y seront acheminés et, par une juxtaposition de techniques d'utilisation de la recherche et de R-D définies et coordonnées, le personnel tentera de trouver rapidement des solutions et d'en alimenter le réseau sous forme de stratégies, de techniques et de règles d'action devenues opérationnelles.

\section{PROCESSUS De ReCherche et dÉVELOPPEMENT : PRINCIPALES PHASES}

Organismes, entreprises et agences gouvernementales proclament littéralement par milliers leur association directe ou indirecte à la R-D. Au début des années 1950, à l'instar des expressions " analyse de systèmes " et " aérospatiale ", la R-D a acquis une certaine respectabilité et un certain prestige auprès des agences et des organisations industrielles, gouvernementales et scientifiques. C'était presque comme si l'intégration d'expressions de ce genre dans la structure même de l'entreprise lui conférait un pouvoir mystique menant directement à l'octroi sans restriction de subventions fédérales. Les compagnies quasi scientifiques et pseudo-scientifiques désireuses d'obtenir des subventions virent donc leur nombre décupler.

L'aboutissement logique et absurde d'un tel état de choses peut être illustré par la pièce Go, Baby, Go, où la Kundel Tool \& Cie devient la Universal Aerospace Systems Research and Development Corporation. L'expression « recherche et développement » a été utilisée avec insouciance et imprécision, mais non sans profit. Au fil des ans, ce manque d'attention et de rigueur s'est installé dans le langage courant à un point tel qu'aujourd'hui très peu de profanes saisissent vraiment le processus et l'estiment à sa juste valeur. C'est ce qui a poussé des experts comme Edward B. Roberts $(1967$, p. 5) à déclarer qu'il arrive trop souvent que la R-D, même pour ceux qui travaillent en ce domaine, "repose pour une large part sur des faits et du folklore plutôt que sur des principes découlant des faits eux-mêmes ". Il déplore le fait que " ses processus soient peu compris et que ses responsables ne puissent bénéficier d'une formation structurée ». De même, Richard E. Schutz affirme que les techniciens qui ont acquis 
une bonne maîtrise des travaux de R-D jusqu'ici ne sont nullement enclins à conceptualiser les composantes méthodologiques de leurs propres activités :

[lls] ne se sont guère préoccupés, par conséquent, de présenter leurs macrostratégies sous une forme facilement reconnaissable pour les professionnels d'une spécialisation donnée ou aisément transmissible à d'autres disciplines comme l'éducation... C'est la méthodologie, non pas les spécialistes, qui permet à des domaines comme l'aérospatiale, l'architecture, les affaires, le génie et la pharmacie de contribuer au perfectionnement de l'enseignement [et des services sociaux] (Schutz, 1979, p. 6).

II recommande vivement à ses lecteurs, en outre, de définir les similitudes existant entre les activités intellectuelles des diverses disciplines qu'il énumère. Leur existence même est corroborée par la possibilité de généraliser certains éléments fondamentaux du processus de R-D sur le plan conceptuel, eu égard à la discipline particulière qui l'emploie.

Dans la conceptualisation de la R-D, il convient de partir des définitions proposées par la National Science Foundation, qu'elle utilise lors de ses enquêtes statistiques en ce domaine. Selon elle, il existe trois champs importants : recherche fondamentale, recherche appliquée que nous appellerons ici « création " pour plus de clarté et pour minimiser le recoupement avec d'autres concepts - et développement. Les définitions sont les suivantes :

\section{Recherche fondamentale}

La recherche fondamentale s'explique surtout par le désir de rechercher la connaissance pour elle-même. Le chercheur n'y est pas contraint d'atteindre des objectifs immédiats et vise par son travail à accroître la compréhension des lois naturelles. Les découvertes sur le plan des connaissances fondamentales entraînent souvent des applications d'ordre général, ou certains concepts scientifiques sont élargis ou révisés et leurs conséquences ont une portée considérable (National Science Foundation, 1969, p. 1).

\section{Recherche appliquée ou création}

Le spécialiste effectue la recherche appliquée tout en visant certaines applications précises : cette recherche peut donc [...] avoir pour 
objet de « traduire " les connaissances existantes en de telles applications... Elle se distingue de la recherche fondamentale du fait qu'elle s'efforce de montrer ou d'indiquer comment entreprendre les étapes expérimentales qui permettront de convertir une idée abstraite en but utile. [Elle] aide l'agence à remplir certaines missions, souvent en tant que précurseur du développement (ibid., p. 15).

La recherche appliquée au sens de création, comme nous l'emploierons ici, est mise en évidence par Knut Holt (1975, p. 11-61), qui la définit comme suit :

La recherche appliquée est la création et l'application systématiques de certaines connaissances grâce aux efforts structurés de personnes travaillant pour un objectif déterminé [pratique] [...] Elle vise en principe à trouver une solution (concept de création) pour la mise sur pied ou pour l'amélioration d'un produit, d'un processus ou d'un matériel.

La troisième définition que donne la National Science Foundation est celle du développement :

\section{Développement}

Dans le développement, les conclusions et la compréhension découlant de la recherche s'orientent vers la production de matériel, d'appareils, de systèmes ou de méthodes utiles, de même que vers la conception et vers l'amélioration des prototypes et des processus. Les activités de développement, tributaires des résultats de la recherche, sous-entendent généralement la mise en œuvre et l'expérimentation d'une composante, d'un produit ou d'un processus au point où il pourra satisfaire certains critères fonctionnels ou économiques particuliers... Elles visent des objectifs très spécifiques et habituellement prévisibles (National Science Foundation, op. cit., p. 19).

Par conséquent, son produit est quelque chose que peut utiliser le consommateur. Son objectif est bien défini et, idéalement, le développement se déroule de façon à pouvoir arriver à sa réalisation optimale.

Dans une étude sur l'utilisation des connaissances en sciences sociales importante et notoire, Ronald G. Havelock (1973) met au point un modèle légèrement différent dans lequel il définit recherche, développement et diffusion. II y introduit un élément clé supplémentaire pour 
la recherche et le développement : la diffusion. Si un produit ou un instrument ou un processus nouveau doit avoir une utilité quelconque, il doit être diffusé - il faut le commercialiser, en faire la promotion - et mis à l'essai par les utilisateurs éventuels. Un produit et la diffusion d'un produit peuvent viser une population globale - consommateurs, auditoires, lecteurs par exemple; sinon, ils pourront s'adresser à un groupement professionnel intermédiaire composé de spécialistes comme des médecins, des techniciens de la radiodiffusion ou des praticiens travaillant dans le domaine des services sociaux, ou encore ils pourront être dirigés subséquemment par leur intermédiaire vers des systèmes cibles plus étendus : groupes de clientèle ou électeurs notamment. Pour illustrer cela, on pourrait se représenter la mise au point de nouveaux médicaments, achetables et utilisables directement par les consommateurs ou transmissibles par les médecins de famille dans l'exercice de leur profession. Aux fins de notre propre analyse de la R-D sociale, nous avons choisi de nous concentrer sur le développement de stratégies d'intervention destinées aux praticiens moyens.

Lorsque Ronald G. Havelock traite de diffusion, il emploie des expressions comme dissémination, distribution, installation, adoption et intégration au sein du système cible. II définit également deux fonctions spécialisées qui ressortissent à la diffusion elle-même : le responsable de la transmission et le responsable de l'adoption. Pour lui, le premier participe à des activités comme la promotion, l'information et la démonstration et le second à des activités concomitantes comme la prise de conscience, l'évaluation et l'installation.

Les concepts de Ronald G. Havelock à l'appui, il est maintenant possible d'ajouter un quatrième élément dans cette définition élémentaire de la R-D :

\section{Diffusion}

Une fois que la connaissance a dépassé cette phase de développement, elle est prête à être produite et diffusée massivement auprès de tous les membres de la société à qui elle pourrait servir (Havelock, 1972, p. 2-42).

On peut considérer les étapes qui précèdent comme les phases fondamentales de tout processus de recherche et développement: 
recherche fondamentale, création (ou recherche appliquée), développement et diffusion. Pareille conception quadridimensionnelle est corroborée par d'autres sources. Edward Ames (1951, p. 370-381), par exemple, propose un modèle analogue, fondé sur une analyse qui prend racine en économique et qui est reliée à de nouveaux processus industriels. Les " résultats " projetés de chacune des " étapes " qu'il a proposées sont fort instructifs :

\begin{tabular}{ll}
\hline \multicolumn{1}{c}{ Étapes } & \multicolumn{1}{c}{ Résultats } \\
\hline Recherche fondamentale & $\begin{array}{l}\text { Résultats obtenus en laboratoire, } \\
\text { hypothèses et théories, formules } \\
\text { et documents de recherche }\end{array}$ \\
Travail d'invention (création) & $\begin{array}{l}\text { Modèles de travail, croquis, } \\
\text { inventions brevetées }\end{array}$ \\
Travail de développement & $\begin{array}{l}\text { Plans, devis, échantillons, } \\
\text { inventions brevetées. }\end{array}$ \\
Équipements et processus & $\begin{array}{l}\text { Diffusion et établissement } \\
\text { d'usines nouveau genre }\end{array}$ \\
\hline
\end{tabular}

En R-D les étapes ne sont pas précises, qu'il s'agisse des définitions ou des fonctions en cause. Cela, H.I. Ansoff (1961, p. 210-211) l'exprime très clairement en ces termes :

La variation considérable du degré d'incertitude cause une difficulté sérieuse lorsqu'il s'agit de différencier nettement la recherche appliquée de ses activités préliminaires et subséquentes. D'une part, il n'est pas rare de trouver des projets de recherche appliquée dont les résultats soient si incertains qu'il faille en fait créer de nouvelles connaissances et qu'on devrait, par conséquent, considérer comme des projets de recherche fondamentale...

D'autre part, il arrive assez souvent de trouver un projet visant le développement d'un produit quelconque étiqueté par inadvertance comme un projet de recherche appliquée. Comme dans le cas qui précède, une telle confusion peut entraîner un très mauvais aiguillage des efforts et des conséquences plus grandes encore. Le problème principal provient du fait que, bien qu'il y ait beaucoup de similitudes entre la recherche appliquée et les activités de développement d'un produit quelconque - elles supposent habituellement la construction d'un outil de travail -, leurs buts diffèrent 
totalement. Dans le premier cas, il ne s'agit que de prouver qu'il existe une solution à un problème particulier; dans le second, il faut mettre en branle certaines étapes successives pouvant mener à la présentation d'un produit aux [utilisateurs] éventuels.

S'appuyant sur les expériences menées dans le cadre des Laboratoires Bell, Mervin J. Kelly (1950, p. 287-301) propose un schème sensiblement plus élargi que le précédent. Ses deux premières étapes sont identiques à celles de notre propre schéma : recherche fondamentale et recherche appliquée (ou création). Cependant, il subdivise la phase du développement en ce qu'on pourrait considérer comme deux sousétapes : développement et création d'une part, et organisation en vue de la fabrication d'autre part. La distinction entre les deux repose sur ce que les spécialistes en ce domaine qualifient souvent de « phase d'introduction " (ou préliminaire) ou " phase de terminaison » (ou avancée) du processus de développement. La phase d'introduction est la phase initiale qui chevauche et se confond avec notre propre notion de création. La phase de terminaison est la phase finale, pouvant se juxtaposer très étroitement à la fabrication et à la diffusion. Dans la diffusion, Mervin J. Kelly englobe certaines activités comme la fabrication, la distribution et l'installation. II ajoute qu'à différentes étapes un certain degré d'autonomie peut être nécessaire. Toutefois, selon le degré d'autonomie atteint, il faudra mettre en place de multiples mécanismes charnières.

De toutes les étapes, le développement est la phase la plus complexe et la plus diversiforme à bien des égards. Voilà pourquoi un certain nombre d'écrivains comme Richard E. Schutz (op. cit.) et d'autres spécialistes du Far West Laboratory for Educational Research and Development (1973) l'ont décrit avec autant d'exhaustivité.

Pour élaborer ce qui, pour eux, est un mouvement général de progression du processus de développement, les membres du personnel du Laboratoire puisèrent dans quelques-uns des différents projets qu'ils avaient réalisés. Ce mouvement et son degré de conformité par rapport à nos propres travaux pourraient être présentés comme suit :

Développement embryonnaire du produit

Expérimentation préliminaire sur le terrain
(II correspond à la formulation de nos premiers principes d'action.)

(Elle correspond à notre programme d'expérimentation pilote.) 
Révision du produit

Principale expérimentation sur le terrain

Révision opérationnelle du produit

Expérimentation opérationnelle sur le terrain
(Elle correspond à notre manuel préliminaire.)

(Elle correspond à notre principal programme d'expérimentation.)

(Elle correspond à la seconde révision de notre manuel.)

(Elle correspond à notre recherche des réactions à notre manuel révisé.)

Selon les membres de l'équipe du Far West Laboratory, ces activités sont suivies d'un ensemble de tâches liées à la diffusion. Ces tâches comportent, entre autres, certaines préparations en vue de la dissémination, de la rédaction d'un rapport final sur l'évaluation de l'expérimentation sur le terrain et de l'implantation de nouveaux produits et de nouvelles techniques dans des milieux cibles étendus. Une telle progression - expérimentation, évaluation, développement, expérimentation - correspond au modèle de base suivi dans nos propres travaux. Notre processus est le suivant : élaboration de nos premiers principes d'action; vérification de ces principes dans un cadre expérimental et mise au point de la première version de notre manuel destinée à guider leur mise en application; examen minutieux au moyen d'une importante expérimentation sur le terrain; révision du manuel à la lumière de cette expérimentation; réactions des utilisateurs éventuels de divers milieux, qu'il s'agisse du monde de l'enseignement ou d'un autre milieu; révision conséquente du manuel, plus approfondie encore, jusqu'à ce qu'il prenne la forme d'un produit final utilisable. Voilà exactement comment en théorie devrait se dérouler le processus de développement en R-D sociale.

\section{MOdèle CONJUGUANT UTILISATION DE LA RECHERCHE ET R-D}

Lorsqu'on pénètre dans l'univers de la R-D sociale, on se sent un peu comme Dorothy qui, au sortir de sa maison, se retrouve au Pays d'Oz : c'est un monde plein d'intérêt et d'excitation, mais presque totalement inconnu. Comme l'héroïne de cette histoire, les spécialistes en R-D sociale ont un énorme besoin qu'on leur ouvre la voie. 
Comme partie intégrante de nos travaux en vue de comprendre le processus de recherche et développement en sciences sociales et de le mettre en application, nous avons élaboré un plan de travail comportant les activités qui nous apparaissent les plus importantes en ce domaine. II s'agit d'un modèle empirique et inductif pour une large part, issu des expériences effectives vécues dans le cadre du Projet d'intervention communautaire, alors que nous tentions de manœuvrer avec les découvertes existantes de la recherche fondamentale, d'une part, et les outils d'intervention pratiques, d'autre part. Selon nous, il résume et structure les concepts discutés jusqu'ici. S'agit-il de la meilleure route à suivre? Cela reste à voir; c'est toutefois une avenue qui s'est avérée fort utile.

Notre plan de travail, que nous avons schématisé, comprend six phases essentielles reliées par cinq étapes opérationnelles (voir page suivante). Nous y avons indiqué au bas les quatre phases fondamentales de la R-D, en vue de démontrer comment s'y imbriquent les nombreux concepts et définitions du processus de recherche et développement qu'on peut trouver dans les ouvrages. L'interpénétration des phases de R-D, d'une part, et les étapes et les phases d'utilisation de la recherche, d'autre part, constituent la principale nouveauté conceptuelle que nous introduisons ici. Pour ceux qui ne connaissent guère nos travaux antérieurs, nous résumerons brièvement notre façon de définir actuel-lement les étapes d'utilisation de la recherche.

Notre modèle a pour point de départ un objectif social ou un problème social qui préoccupe un groupe ou un organisme donné, spécialisé en assistance sociale ou dans la distribution des services sociaux. Vient ensuite l'examen de l'ensemble des connaissances empiriques qui existent en sciences sociales comme source de solutions éventuelles au problème lui-même ou comme indication possible de moyens de réalisation de l'objectif social. Le modèle suit une longue progression jusqu'à l'étape ultime : une utilisation généralisée chez les professionnels des services sociaux. Chacune des cinq étapes opérationnelles, que nous avons insérée à l'intérieur d'une flèche en raison de son rôle plus actif, se transforme ensuite en une étape essentielle : un produit repère.

De tels produits peuvent être multiformes : expériences, données, mécanismes, manuels de procédures, manuels didactiques, etc. Chaque étape essentielle ainsi que ses produits, que nous avons représentés 
Schéma du processus d'utilisation de la recherche et de la R-D

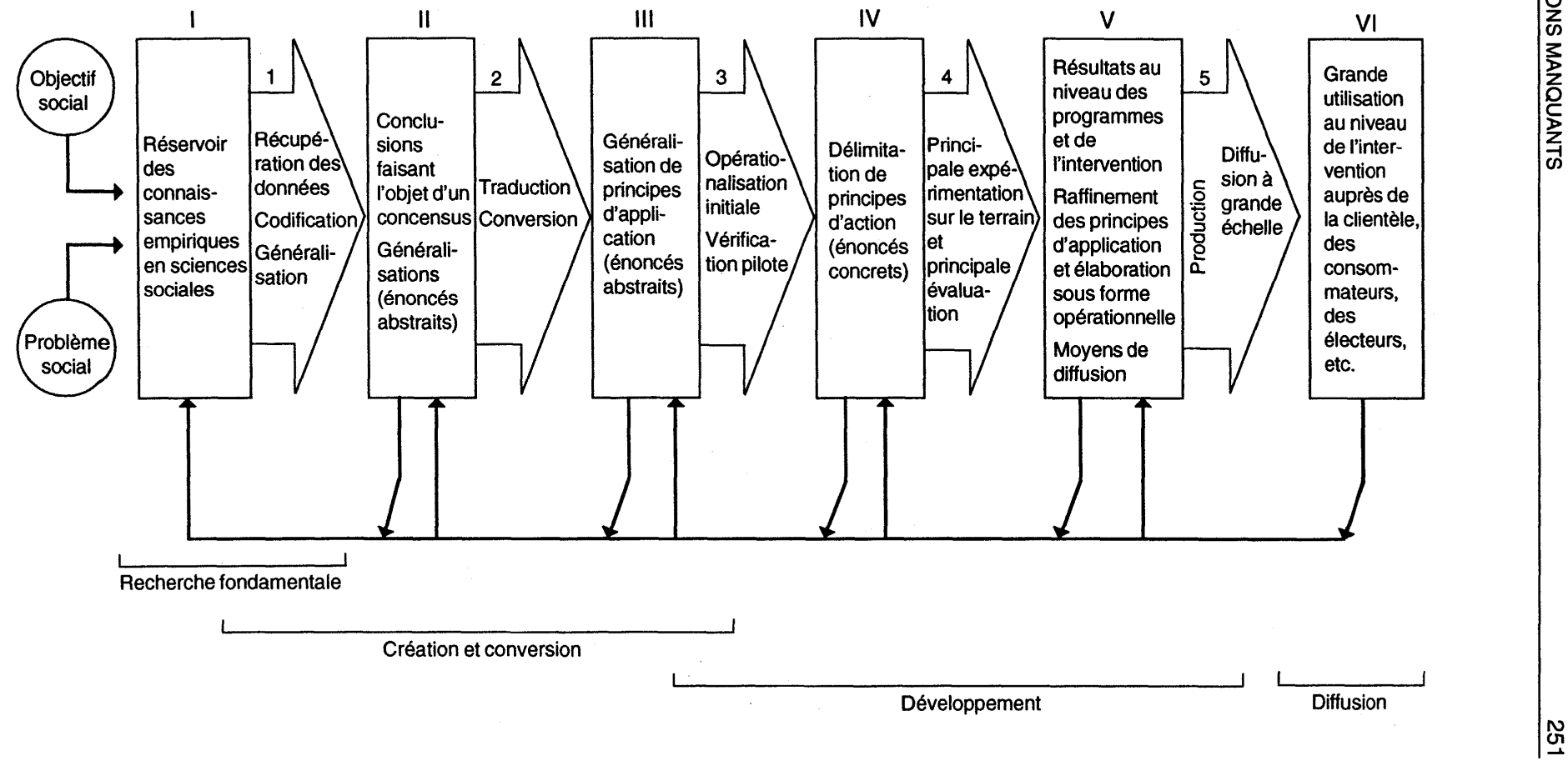


par une case sur notre modèle, mènent à leur tour à l'étape suivante et lui servent de fondement. Dans la description quelque peu détaillée de chacune des phases essentielles et de chacune des étapes opérationnelles subséquentes, il sera utile de se reporter au schéma (p. 251). II sera ainsi possible de ne pas perdre de vue la position de chacune des diverses composantes dans l'ensemble du modèle.

\section{Phase essentielle I}

Principale source de connaissances au niveau de la recherche en sciences sociales

II s'agit d'une source constituée par le réservoir des données empiriques existantes, disponibles dans les ouvrages officiels des spécialités et des professions. Cela englobe aussi d'autres sources moins officielles : rapports des agences, exposés et mémoires rédigés dans le cadre de projets. Certaines font l'objet d'une publication indépendante et d'autres apparaissent dans des répertoires ou dans des fichiers de divers types. D'autres sont à la fois mentionnées et abrégées dans des répertoires ou dans des fichiers centraux. La catégorie d'ouvrages et le type de données seront choisis pour examen minutieux selon le type d'objectif social ou de problème social visé par le processus de R-D sociale - ou les deux conjointement.

\section{Étape opérationnelle I}

Récupération de données, codification, généralisation

- Cette étape nécessite le repérage de sources valables où l'on pourra obtenir des données pouvant servir à la recherche en ce qui regarde la présentation de problèmes d'intervention ou d'objectifs. L'utilisation de sources tant primaires que secondaires est possible, de même que l'emploi de réservoirs d'information sur mémoire.

- Les études portant sur le problème ou sur la question à examiner doivent être choisies à partir du réservoir d'origine. Certains problèmes de nomenclature se posent ici, de même que celui d'un choix judicieux de descripteurs. II faut ensuite surmonter certaines 
difficultés inhérentes à l'hétérogénéité taxinomique de la connaissance en sciences sociales et en intervention sociale. Les différentes perspectives d'intervention doivent ordinairement se traduire en éléments analogiques de type scientifico-linguistique pour qu'on puisse tirer parti des sources scientifiques et les reconvertir par la suite.

- L'évaluation des données doit se faire suivant leur degré de solidité, de validité et d'applicabilité.

- Leur codification doit ensuite s'effectuer d'après des catégories de connaissances appropriées.

- Divers travaux doivent être faits en vue de découvrir une certaine homogénéité dans les conclusions et de reconnaître une certaine uniformité dans les données choisies.

- Certaines généralisations et certaines propositions doivent parfois être formulées à partir des conclusions homogènes.

\section{Phase essentielle II}

Conclusions homogènes, généralisations et propositions

Comme semble le sous-entendre la description qui précède, le produit consiste à ce stade-ci en une ou plusieurs généralisations qui reposent sur la reconnaissance de certaines lois ou de certains principes scientifiques ou sur la convergence de quelques conclusions hétérogènes découlant d'études axées sur la recherche. Lorsqu'il y a concordance entre diverses études menées en de multiples occasions et dont les thèmes et les méthodologies diffèrent, on peut alors davantage se fier à la validité de ces généralisations. Tel est particulièrement le cas lorsque les découvertes générant des conclusions contradictoires sont rares - sinon inexistantes.

En raison de leur tendance à synthétiser diverses études et à aboutir à une certaine homogénéité scientifique, les généralisations seront abstraites. Elles tendront aussi à décrire des phénomènes sociaux plutôt qu'à fournir des prescriptions sur l'intervention. Un tel état de choses n'est guère surprenant, puisque la recherche en sciences sociales s'efforce largement de décrire et de comprendre la société, non pas de la transformer. 
Les conclusions homogènes peuvent décrire un simple état d'uniformité sur le plan empirique ou consister en propositions descriptives d'une relation cohérente et suivie entre deux variables au moins.

\section{Étape opérationnelle II}

\section{Conversion et création}

À ce stade-ci, la tâche essentielle consiste en une application sur le plan cognitif :

- Les traductions doivent maintenant se faire d'un langage scientifique vers un langage qui convient mieux à un type d'utilisation normale dans le cadre des situations d'application.

- La conversion d'une formulation descriptive en une formulation normative peut créer une « interruption sur le plan déductif » par l'écart qui existe entre la généralisation et le principe d'intervention. Ce sont là les dimensions création et " phase d'introduction » du développement de la R-D. II faut prendre en considération l'importance des interruptions déductives et la direction de la mise en application (en vue d'arriver à la stabilisation ou au changement organisationnel, par exemple).

- Les différents aspects de la " réalité " à l'étude doivent alors être soupesés : praticabilité ou possibilités d'implantation d'une application donnée, coût, conditions limitatives et éléments restrictifs ou utilisation d'un canal quelconque.

\section{Phase essentielle III}

\section{Grands principes d'application}

Les résultats du processus de traduction-conversion-création se situent ici. À ce stade-ci, la formulation normative - que nous appellerons Grands principes d'action - représente certains produits. Alors qu'ils sont maintenant d'une utilité plus immédiate pour les praticiens, ces énoncés normatifs découlent de grandes généralisations ayant une certaine homogénéité. De plus, leur forme tend à être plutôt abstraite. L'implantation concrète des principes d'application y est laissée à la créativité des praticiens à moins que le processus d'utilisation ne 
soit poussé plus avant. Au niveau de la R-D industrielle, on peut produire un modèle assez rigoureux, corroboré par des données mathématiques, dont la viabilité et la praticabilité sur le plan économique restent encore à démontrer.

\section{Étape opérationnelle III}

\section{Opérationalisation initiale et expérimentation témoin}

L'opérationalisation en vue de l'implantation suppose une description plus limitée des localisations, des contextes, du matériel, des ressources et des comportements. Le type d'agence et le type de personne touchés par l'application elle-même en sont deux exemples - il s'agit plus généralement des référents empiriques pour tous les éléments du principe d'application.

L'opérationalisation peut être une fonction cognitive-perspective partiellement, mais elle doit englober une implantation expérimentale concrète ou une certaine mise en œuvre qui permettra la vérification et l'élaboration de normes empiriques. Pour cela, il faut effectuer une expérimentation témoin dans le cadre d'implantation projeté ou en employant son matériel, ses mécanismes et ses acteurs. Le travail expérimental s'impose partiellement pour déterminer l'efficacité du concept de création dans le cadre concret. Le praticien agit au niveau de la phase d'introduction d'un rôle d'ingénierie sociale.

\section{Phase essentielle IV}

Délimitation des principes d'application

L'opérationalisation initiale entraîne une démonstration beaucoup plus exhaustive et beaucoup plus circonscrite des principes d'application. Elle peut supposer une concrétisation accrue de chacun des éléments définis comme une composante clé du principe d'application. Sous cette forme, on peut faire l'analyse de l'application dans des centres de santé mentale en comparant ceux-ci à des bureaux d'urbanisme, dans des grandes agglomérations en les comparant à des petites, dans des agences à gros budgets en les comparant à des agences limitées financièrement, etc. En outre, certains protagonistes, certaines lignes d'action ou certaines ressources peuvent y être indiqués. 
Par la structuration du concept de création sous certaines formes opérationnelles, cette première expérimentation engendre certains schémas de travail et certains prototypes préliminaires. Pareille façon d'opérationnaliser l'application guide bien davantage le praticien et lui accorde une certaine confiance en la viabilité de la formulation de l'application elle-même. Dans le cadre de notre travail, cette étape a résulté en la version préliminaire du manuel des praticiens.

\section{Étape opérationnelle IV}

Implantation plus complète et principale expérimentation sur le terrain

- Cette étape suppose l'existence d'un manuel de procédures préliminaires, de documents de travail, d'équipements audiovisuels, etc., pouvant être mis à la disposition des techniciens du développement. On attend d'eux qu'ils opérationnalisent le concept de création avec une certaine exhaustivité et une certaine permanence.

- La principale étude intégrale sur le terrain a pour objet de déterminer si le matériel sera utilisable par un vaste échantillon représentatif de praticiens utilisateurs.

On cherche aussi à y modifier le concept de création, à l'élargir, à l'opérationnaliser davantage et à évaluer son efficacité, à la lumière des résultats projetés auprès de la clientèle, des consommateurs, etc. Cette vérification de l'efficacité par une évaluation des résultats rigoureuse revêt une importance particulière. Elle permet d'esquisser des prescriptions assez solides quant aux conditions, aux contraintes et aux mécanismes de mise en application et englobe certains éléments de recherche évaluative en les plaçant dans un contexte plus productif de R-D. Pareille forme de vérification de la réalité peut produire des effets rétroactifs qui modifient le principe d'action et peut même transformer la théorie fondamentale l'ayant engendré.

- Les tâches sur le terrain comprennent, entre autres, le choix d'emplacements ou d'utilisateurs, le recrutement et la formation d'experts en ingénierie sociale, l'utilisation de stimulants, la résolution de problèmes d'entrée, la clarification du rôle des praticiens sur le terrain et sa relation face au rôle du spécialiste en recherche appliquée. 
- Les tâches de recherche englobent la désignation d'une méthodologie appropriée pour l'étude et pour le perfectionnement des instruments, la mise en œuvre, l'adaptation à des problèmes spéciaux comme les résistances entre praticien et technicien ou les effets de Hawthorne, l'évaluation de l'efficacité et la détermination de critères de rejet, de modification ou d'authentification du concept de création.

\section{Phase essentielle V}

Résultats au niveau de l'intervention et des programmes; vérification, raffinement et élaboration de concepts de création; moyens de diffusion

À ce stade-ci, on devrait avoir créé plusieurs produits utiles. Tout d'abord, il est maintenant possible de déterminer les effets du concept opérationnel de création lorsqu'il est mis en application par un échantillon représentatif d'utilisateurs réels. Pareil type d'évaluation peut se faire au moyen de mécanismes de recherche formels ou par des méthodes d'observation et d'évaluation moins orthodoxes. Le produit de la principale expérimentation sur le terrain fait habituellement l'objet d'une autre vérification de la part des utilisateurs éventuels en vue d'atteindre son perfectionnement optimal et de mettre davantage au point l'instrument, le processus ou encore le produit lui-même. Cette étape peut aussi faire ressortir certains problèmes et certaines possibilités qu'il faudra prendre en considération à la phase de diffusion subséquente.

Enfin, le matériel de diffusion peut être cristallisé, englobant les résultats des travaux sur le terrain : manuels ou guides du praticien, bandes magnétiques pour enregistrement, bandes magnétoscopiques, documents, graphiques, etc.

Les effets rétroactifs des phases IV et $V$ peuvent être lourds et intermittents, et on peut observer un certain nombre de cycles d'essai, de modifications suivies d'autres essais. Le caractère cyclique du développement est particulièrement marqué. Aussi la nouvelle version révisée du guide des praticiens fut-elle évaluée de manière plus exhaustive par les praticiens eux-mêmes dans le cadre de milieux d'application. 


\section{Étape opérationnelle $\mathbf{V}$}

\section{Production et diffusion étendue}

Cette étape comprend les éléments qui suivent :

- Reproduction massive de médias comportant certaines stratégies de solution opérationnalisées : guides, manuels, graphiques, formulaires, etc. La production est habituellement confiée à une imprimerie ou à une maison de publicité expérimentée et disposant des ressources pour mener cette tâche technique à bonne fin. Dans le cadre d'une version élargie de la R-D, la production peut être considérée comme une phase distincte, comme c'est le cas en R-D industrielle.

- Identification, en tant qu'utilisateurs cibles éventuels, d'un groupe de praticiens ou d'organismes donnés. Pareille démarche devrait d'abord se faire au début du processus de R-D, mais à ce stadeci elle doit être précisée davantage et évaluée à nouveau.

- Détermination de leurs caractéristiques, de leurs attitudes et de leurs besoins. II s'agit là essentiellement d'une forme de recherche commerciale.

- Choix de mécanismes d'information et de publicité appropriés et présentation plus intéressante et plus exacte du matériel.

- Information et motivation des utilisateurs éventuels.

- Repérage des responsables fonctionnels, des prescripteurs ou des réseaux de professionnels non officiels en vue de les utiliser comme canaux de diffusion.

- Formation et renforcement préliminaires possibles des utilisateurs.

- Mise au point de mécanismes de scrutation des résultats d'une application plus grande, etc.

\section{Phase essentielle VI}

Grande utilisation dans la pratique

Le processus devrait se terminer par la généralisation de l'utilisation du principe d'application mis au point sur le terrain. II devrait alors 
avoir subi l'expérimentation avec succès, et on devrait avoir développé certains moyens de renseigner efficacement les praticiens sur la meilleure façon de le mettre en œuvre. Les bénéficiaires éventuels sont les clients, les consommateurs et certains éléments du réseau des services sociaux. Une plus grande expérimentation auprès de la clientèle, tant sur le plan de la méthode que sur le plan des résultats, devrait encore hypothétiquement alimenter rétroactivement le processus global, laissant entrevoir les modifications et les raffinements qui s'imposent à chacune des étapes, ce qui peut même aller jusqu'à remonter à la phase de recherche fondamentale.

Il est tout naturel pour nous d'avoir centré ce processus de R-D sur les travaux que nous avons effectués dans le cadre du Projet d'intervention communautaire et de l'avoir illustré par nos propres expériences. Cependant, on pourrait remplacer l'expression « praticien-utilisateur » par " gestionnaire-utilisateur », " professeur-utilisateur », " politicienutilisateur », « syndicaliste-utilisateur » ou par tout autre applicateur virtuel d'une recherche fondamentale en sciences sociales, opérationnalisée grâce à un processus de R-D sociale.

La synchronisation entre les quatre principales composantes du processus de R-D et le schéma d'utilisation de la recherche se fait comme suit :

Recherche fondamentale Phase I, peut-être aussi Étape I et Phase II

Conversion et création (recherche appliquée)

Développement

Chevauchement possible de l'Étape I et de la Phase II Concentration sur l'Étape II et sur la Phase III

Recoupement possible avec l'Étape III

Étape III jusqu'à la Phase V inclusivement

Diffusion

Étape V et Phase VI

Il importe ici de faire une certaine mise au point concernant la juxtaposition du schéma d'utilisation de la recherche et de la R-D. Notre travail initial dans le cadre du Projet d'intervention communautaire a porté sur le développement, sur l'expérimentation sur le terrain et sur la diffusion de normes destinées aux praticiens des services sociaux. Nous n'avons pas vérifié les hypothèses au sens traditionnellement reconnu; les généralisations à la base de nos principes d'action avaient déjà été établies. Aussi n'était-il nullement question de « prouver » ou de « réfuter " les principes d'action conséquents. La démarche que nous avions choisie visait plutôt l'énonciation efficace et opérationnelle de ces principes. C'est là la dimension développement du processus de R-D. Le travail sur le terrain par une vérification évaluative 
du rendement indique quel est le degré d'efficacité de l'application et dans quelles circonstances elle se fait. II en résulte donc un instrument de travail plutôt qu'un principe scientifique.

Toutes les conceptions de R-D ne requièrent pas une démarche comportant des étapes et des composantes du type de celles que nous avons représentées dans la dynamique du processus étudié. Au moins deux autres points de vue restent à découvrir. Dans le premier cas, on considère la R-D sur le plan de la mise en œuvre des systèmes et de l'organisation scientifique du travail. On y fait reposer sur la rationalité et sur l'efficacité la réalisation d'objectifs dans un milieu où s'accomplissent des tâches techniques complexes (Frey et Goldman, 1967; Hall, 1962; Kappel, 1976). On qualifie la méthode par l'emploi d'expressions comme "technologie créative structurée " (Ames, 1951) et on considère qu'elle permet « d'améliorer la qualité, la fidélité et la cohérence du produit final ", tout en diminuant le coût de la production (Frey et Goldman, 1967).

Le second point de vue est celui des principaux collaborateurs de la revue Educational Technology. L'importance y est accordée à la production de médias éducatifs, grâce à une planification rigoureuse et à une vérification sur place auprès du groupe de consommateurs visé. La démarche intellectuelle consiste en une théorie d'apprentissage ou en une modification du comportement, et le contexte est un cadre d'enseignement valable, évalué sur le plan empirique - il ne s'agit pas nécessairement de la conversion délibérée de certaines connaissances scientifiques essentielles en programmes et en instruments opérationnels. Dans pareil contexte, c'est la théorie d'apprentissage qui est véhiculée et mise en application. La R-D éducationnelle suit ce schème dans une large mesure.

En faisant certaines observations sur le mode opératoire suivi habituellement dans l'élaboration des programmes sociaux, Amitai Etzioni (1976, p. 11) fait ressortir l'absence d'une planification et d'un processus de développement systématiques et progressifs, comme ceux que nous venons de décrire :

Les programmes sociaux mis au point dans les années 60 , les campagnes contre la toxicomanie et contre le crime, les luttes contre le cancer et le goût récent de découvrir de nouvelles ressources énergétiques nous ont appris que, alors que nous tentions d'utiliser les ressources nationales pour résoudre nos problèmes 
sociaux, nous avions entrepris des projets extrêmement ambitieux, sans connaissances fondamentales suffisantes ou sans le soutien nécessaire. Des programmes de plusieurs milliards de dollars, conçus à Washington, D.C., ont été implantés à partir d'éléments théoriques qui n'avaient guère fait l'objet de vérifications ou de recherches préalables. Surles plans humain et financier, nous ne pouvons nous permettre le gâchis onéreux que cela occasionne. II nous faut donc moins de programmes gouvernementaux, mais que ceux que nous aurons soient mieux décortiqués ou plus perfectionnés.

Etzioni poursuit en recommandant une démarche faisant l'intégration de bon nombre de caractéristiques fondamentales du modèle mixte d'utilisation de la recherche et de R-D sociale que nous avons élaboré dans le présent exposé. II s'exprime en ces termes :

Dans la construction de nouveaux vaisseaux spatiaux, de nouveaux armements, de nouveaux avions, voire de nouveaux jouets, il est assez courant de partir systématiquement d'un concept théorique pour en arriver à une ébauche sur papier, à un modèle réduit assujetti à divers tests, jusqu'à la production d'un prototype grandeur nature ou de plusieurs prototypes de ce genre: tout cela, avant même que la production en série ne soit autorisée. En général, certaines modifications sont faites à chacune des étapes à la lumière de l'expérience, et ce, précisément parce qu'en règle générale on ne peut anticiper ou rationaliser toutes les réactions et tous les développements. À l'instar des technologies nouvelles, les nouveautés au niveau des programmes gouvernementaux [et certaines tentatives de solution d'un problème social] se doivent de passer par un processus de R-D complet aussi bien que rigoureux (Etzoni, 1976, p. 11).

\section{RECHERCHE ET DÉVELOPPEMENT : UTILITÉ EN SERVICE SOCIAL}

Chercheurs et hommes d'action s'admonestent réciproquement, en quelque sorte, pour les échecs dans la communication et pour les déficiences dans la mise en application. Ces admonestations ad hominem découlent d'une inaptitude à reconnaître qu'il s'agit là d'un problème structural avant tout. II n'existe aucune technologie charnière ni aucun instrument intermédiaire pour rattacher la théorie sociale à l'intervention sociale. Dans le domaine social, le vaste réseau complexe des rôles techniques et des institutions qui caractérise les sciences 
physiques est totalement absent. C'est comme s'attendre à ce qu'un théoricien spécialisé en physique de Princeton énonce ses propositions et ses théorèmes sous une forme immédiatement utilisable par le chef d'équipe d'une usine au New Jersey.

La principale conclusion à tirer d'une telle analyse est que le service social et d'autres professions de services individualisés devraient en venir à développer certains mécanismes techniques : institutionnalisation de rôles charnières et de structures de divers types qui serviraient à faciliter la transition entre les connaissances sur le plan de la recherche, d'une part, et les programmes et les interventions au niveau de la société, d'autre part. Dans son analyse des Laboratoires Bell, Egon G. Guba, spécialiste en R-D éducationnelle, fait mention de certaines particularités fondamentales d'un tel modèle institutionnel :

Dans sa grande sagesse, l'A.T. \& T. ${ }^{4}$ a intercalé une vaste organisation entre les producteurs de connaissances et les consommateurs ultimes. Ce réseau, la Western Electric, a son propre cercle de techniciens, qui se répartissent eux-mêmes en diverses spécialités. Certains sont responsables de la mise en application des prototypes; d'autres s'occupent de les mettre à l'essai et de les perfectionner. D'autres encore voient à concevoir les applications éventuelles de façon à rendre leur production possible et économique. Enfin, bien sûr, les spécialistes en production font la production effective des appareils qui seront installés et utilisés par les compagnies de Bell Telephone.

Egon G. Guba souligne l'absence d'éléments équivalents et de mécanismes de conversion dans les disciplines sociales, ce qui entraîne des blocages et des déficiences au niveau de l'utilisation de la recherche. Une telle imperfection passe inaperçue au sein de l'organisation professionnelle même. Au contraire, le personnel tend

à combler le fossé entre l'intervention et la recherche en l'imputant au peu de collaboration entre les chercheurs ou à l'indolence et à l'ignorance des praticiens, ou à l'un et à l'autre à la fois.

Quand on considère la situation sous cet angle, il devient évident qu'on peut percevoir non pas un seul mais de multiples écarts en sciences sociales et que de nombreuses charnières sont laissées pour compte. La structuration même de la recherche au sein des professions de

4. Note du traducteur : American Telephone \& Telegraph Co. 
services individualisés n'amoindrit aucunement le problème. En service social et dans d'autres disciplines, les universitaires et les étudiants du doctorat accumulent des connaissances, et il arrive trop souvent qu'ils fassent part de leurs résultats d'une façon globale à d'autres universitaires, ne laissant que rarement entrevoir certaines implications que pourraient avoir leurs découvertes sur le plan des activités d'intervention et sur le plan des programmes, même sous forme rudimentaire.

Les divers types d'agences constituent une autre voie de production de la recherche. On y fait surtout de la recherche opérationnelle ou de l'évaluation de programmes, études où l'on traite de façon restreinte d'un problème particulier sur le terrain et de sa solution dans un contexte donné. Ces études sont le reflet d'un certain empirisme de clocher, car elles ne sont guère généralisables au-delà de la situation ellemême habituellement. En outre, dans nombre de cas, on puise peu dans la théorie et dans les connaissances en sciences sociales pour clarifier les travaux de recherche. On fait usage des techniques de recherche sociale et non des connaissances importantes qu'elles ont permis d'accumuler.

La recherche parrainée par les organismes gouvernementaux prend la même tangente. D'une part, certains services comme la National Science Foundation et le National Institute of Mental Health incitent les spécialistes à faire de la recherche fondamentale ou " pure " en leur accordant des bourses. D'autre part, les agences en activité, comme la HEW et la HUD, se fient de plus en plus sur les RFP et sur les contrats, où les prescriptions sont esquissées rigoureusement en fonction de certains problèmes opérationnels concrets et adaptées en ce sens. En tentant de contrecarrer une tendance antérieure où l'on permettrait la réalisation d'études théoriques trop vagues et trop abstruses, ces agences se sont engagées dans un mouvement de balancier qui les conduit vers des études appliquées trop rigides.

Comme solution possible, il faudrait accorder plus d'importance aux étapes qui, au niveau d'une agence, se situent entre la recherche fondamentale et les activités d'intervention : le domaine de la conversion, de la jonction et de la mise en application. Pour faire quelque chose en ce sens, il faudrait déterminer une certaine catégorie de rôles charnières, de mécanismes et de modes d'organisation, ce qui impliquerait aussi une certaine interpénétration et un certain fusionnement des intérêts et des compétences sur les plans scientifique et 
appliqué. II s'agit là d'une question très vaste que nous ne pouvons qu'effleurer ici.

\section{Rôles charnières et mécanismes}

L'examen rétrospectif du schéma d'utilisation de la recherche permet de voir un certain nombre de rôles charnières qui en sont conséquents, parmi lesquels on peut citer :

- les spécialistes en récupération de données;

- les spécialistes en conception de politiques et de programmes;

- le personnel de développement : responsables de l'implantation de programmes, chercheurs au niveau du développement, techniciens de la vérification des résultats;

- les responsables de la création de moyens de diffusion;

- les experts en diffusion.

Certains de ces rôles existent actuellement à un degré quelconque, mais ils ne sont pas aussi précis, aussi organisés et aussi interdépendants que ceux que nous avons proposés. Par exemple, dans les services sociaux, on peut trouver des spécialistes en information ainsi que des experts en diffusion qui ont pour tâche de former certaines personnes et de faire connaître certains ouvrages. En fait, les vendeurs des maisons d'édition sont parmi les vulgarisateurs des découvertes et des produits en sciences sociales les plus actifs. Certains rôles sont toutefois extrêmement rares actuellement : les spécialistes en conversion et en création, surtout, ainsi que l'ensemble impressionnant de fonctions rattachées au processus de développement. À l'extérieur de la R-D éducationnelle, le développement est presque inconnu en sciences sociales, selon l'acceptation que nous lui donnons ici.

Le rôle de " spécialiste en utilisation de la recherche " est une fonction intéressante et virtuellement utile. Il a été institué dans le domaine de la réadaptation professionnelle, et Edward $\mathrm{M}$. Glaser et Thomas $\mathrm{E}$. Backer (1975, p. 388) l'ont soumis à une étude d'évaluation. Suivant ces auteurs :

Le spécialiste en utilisation de la recherche avait pour tâche de modifier le fonctionnement de l'agence de réadaptation de l'État 
pour en arriver à une amélioration de la distribution des services soutenue et valable... II avait pour mission de bonifier le climat régnant au sein d'un organisme donné où de nouvelles connaissances pourraient vraisemblablement être mises en application.

Parmi les fonctions énumérées, on pouvait englober le dépouillement des connaissances, la réalisation de projets spéciaux, la consultation pour le personnel de l'agence, la tenue de conférences et d'ateliers et la diffusion de données. Edward M. Glaser et Thomas E. Backer ont fait ressortir les points forts et les points faibles de ce nouveau rôle et ont proposé certains critères de réussite.

Le spécialiste en utilisation de la recherche est un agent de mise en $œ u v r e$ de la recherche au sein d'une agence en activité. Le spécialiste en vulgarisation est une variante de ce rôle et ses activités s'étendent d'une agence initiatrice de la diffusion des connaissances à d'autres organismes au sein de la collectivité et au grand public. Une telle reformulation du rôle de l'agent de vulgarisation agricole a été préconisée par David L. Clark (1962, p. 105-127) pour l'éducation, par exemple.

Pour ce qui est des mécanismes charnières, nombreux sont les organismes spécialisés dans la lutte contre la pauvreté (Crisswell, 1969, p. 711), dans la réadaptation professionnelle (Department of Guidance and Psychological Services, 1968) et dans le contrôle de la pollution (Crowe et Madancy, 1974) qui ont proposé et qui ont utilisé un congrès sur l'utilisation de la recherche quelconque. On a donc effectué certaines études en vue de découvrir des moyens plus efficaces pour que ce type de congrès serve à sensibiliser praticiens et responsables des programmes aux conclusions issues de la recherche et les poussent à les utiliser. Le projet « projet de séminaire itinérant 》 est une variante de ce type de congrès utilisée par la System Development Corporation (Carter, 1968).

Le Service de protection de l'environnement ${ }^{5}$ fait état de nombreux moyens de communication de masse qui lui ont servi à promouvoir certains travaux axés sur la recherche : manuels de conception, rapports techniques abrégés, publications issues de réunions d'information,

5. Note du traducteur : Environmental Protection Agency. 
guides, plaquettes explicatives de processus et de projets, notes d'information, bandes magnétoscopiques et films (Crowe et Madancy, op. cit.).

II existe d'autres rôles et d'autres processus charnières qui ont été employés ou qu'on peut envisager. Ces quelques illustrations devraient toutefois suffire à donner un aperçu des possibilités.

\section{Organismes charnières}

Plusieurs des fonctions et des mécanismes que nous venons de préciser peuvent s'intégrer dans les organisations existantes. Le spécialiste en information par exemple peut faire partie du programme d'éducation permanente d'une école de service social (Wolf, 1976, p. 8-9) ou d'un service spécialisé d'une agence sociale (Research Utilization Inventory, 1975-1976). Par contre, il serait souhaitable de créer de nouveaux types d'organisations centrées sur la R-D, où il y aurait recoupement de plusieurs des rôles et des mécanismes préalablement décrits, sinon de tous. En sciences physiques et dans l'industrie, une telle organisation spécialisée est représentée par le laboratoire de R-D. La création d'organisations analogues dans le domaine des services sociaux semblerait recommandable et judicieuse. Celles-ci pourraient s'intégrer dans de vastes réseaux de distribution de services reliés à la santé mentale, au vieillissement, à la protection de l'enfance, etc., sinon se rattacher aux écoles de service social.

Dans une publication antérieure, nous avons laissé entrevoir la possibilité de conférer aux écoles de service social de niveau postérieur au premier diplôme et à d'autres institutions axées sur les services sociaux un statut exceptionnel en tant qu'institutions charnières; nous avons également souligné le fait que les centres et les instituts de recherche au sein des écoles professionnelles convenaient particulièrement bien comme pivots sur le plan des connaissances. Pareil point de vue est également sanctionné dans un des rapports de la National Science Foundation où l'on affirme :

Les professions - le droit, la médecine, le génie, le service social, le journalisme, les disciplines psychiatriques et la pédagogie représentent quelques-unes des principales institutions sociales pouvant servir à traduire les connaissances en sciences sociales en mécanismes d'intervention utilisables quotidiennement [...] Les 
professions ont un rôle distinctif à jouer dans la traduction des connaissances en actions concrètes (National Science Foundation, 1969).

Dans les écoles de service social, un tel état de choses est largement imputable à la structuration même de l'enseignement. Les facultés comptent dans leurs rangs des instructeurs responsables de l'enseignement des techniques d'intervention aussi bien que de celui des sciences sociales fondamentales et de la recherche. Au sein d'une école, il est tout à fait vrai qu'un département donné - par nécessité peut-être - puisse définir sa mission selon des normes rigoureuses, sinon étriquées et statiques. Néanmoins, l'école de service social dans son ensemble présente d'excellentes occasions d'échanges réciproques entre les mondes universitaire et professionnel, ce qui en fait une structure pleine de possibilités pour la promotion de l'utilisation de la recherche. Sa concentration sur les problèmes humains et sociaux - plutôt que sur la transmission des éléments cognitifs traditionnels inhérents aux disciplines elles-mêmes - lui permet de transcender ces normes. Il est donc plutôt déconcertant que de telles possibilités ne soient pas exploitées plus à fond. Nous ne profitons pas intégralement de tout ce que ce milieu professionnel peut offrir pour la solution de problème interdisciplinaire, avec tous les avantages que cela peut impliquer.

En travaillant dans le champ interstitiel, il est important pour les nouvelles organisations axées sur l'utilisation de la recherche de considérer deux facteurs. II s'agit tout d'abord de la juxtaposition nécessaire de diverses disciplines : spécialités scientifiques et disciplines d'intervention. Une telle interdépendance de compétences et de points de vue peut causer des frictions, mais elle assure la multiplicité sur le plan des perspectives qui s'impose pour une utilisation de la recherche efficace. En comparant un certain nombre de schémas d'utilisation, Maurice J. Eash (1968, p. 410-418) conclut que

le modèle de coparticipation, où chercheur et praticien sont liés, constitue le meilleur moyen de stimuler l'utilisation de la recherche dans le monde de l'éducation.

Une étude menée par James L. Price sur un thème assez différent semble corroborer davantage l'importance d'une telle coparticipation. Celui-ci s'est rendu compte que la Oregon Game Commission avait 
obtenu plus de succès dans l'introduction de nouvelles connaissances en gestion de la faune que n'en avait obtenu la Oregon Fish Commission, dont le personnel était composé surtout de chercheurs diplômés universitaires en biologie. Les préposés à l'appareil à éclosion qu'elle desservait s'en distanciaient considérablement sur le plan social, ses chercheurs étaient moins respectés et leurs découvertes étaient reconnues et utilisées avec une plus grande résistance. La Oregon Game Commission était généralement mieux structurée et comptait dans ses rangs des spécialistes orientés à la fois vers la recherche " pure » et vers la recherche appliquée. Les gardes-chasse qu'elle desservait s'en distanciaient beaucoup moins sur le plan social et étaient, en général, plus enclins à accepter et à utiliser ses découvertes (Price, 1964).

Le second facteur clé qu'il faut considérer est l'importance de l'application, dans des milieux expérimentaux - se rapprochant le plus possible de ceux des utilisateurs éventuels -, des différents mécanismes de recherche inhérents au développement. Cela signifie donc le déplacement de l'appareil de recherche hors du laboratoire et de l'université jusque dans l'univers de l'intervention sociale. C'est ce que Gerald Gordon et Sue Marquis (1966, p. 195-202) ont corroboré dans une étude où un comité composé de sociologues de la santé bien connus a évalué le degré de nouveauté de certaines ébauches de projets soumis par près de deux cent cinquante directeurs de programmes et entrepris dans certaines universités, certains organismes de santé, certaines écoles de médecine, certains hôpitaux et certaines cliniques. Selon eux, non seulement la recherche menée dans des cadres expérimentaux avait-elle tendance à être plus innovatrice que celle des milieux purement universitaires, mais elle pouvait aussi être évaluée plus facilement et adaptée plus aisément.

Tout récemment, nous avons fait certaines propositions quant aux critères structuraux nécessaires aux organismes spécialisés en R-D où existent les compétences requises sur le plan interdisciplinaire et le milieu expérimental que nous venons d'analyser - il s'agit surtout d'institutions qui mettent en œuvre des programmes de R-D complexes (Rothman, 1977). II ne convient pas ici d'approfondir un tel sujet : nous nous contenterons plutôt de souligner succinctement certaines 
implications éventuelles de la présente étude pour les activités organisationnelles du Council on Social Work Education (CSWE) et de la National Association of Social Workers (NASW).

Selon nous, le CSWE devrait davantage promouvoir l'enseignement de certains des rôles charnières et des fonctions d'ingénierie sociale préalablement énumérés. Ils sont difficiles à transmettre, nous le reconnaissons, et cela parce qu'ils excèdent ou chevauchent les disciplines et professions existantes. II est néanmoins possible de faire preuve d'un certain degré de prévoyance et de créativité dans la relance d'une telle formation. Cela suppose en partie l'intensification de la collaboration entre les professeurs de sciences sociales spécialisés dans de nombreuses disciplines et peut aussi signifier certains cours placés conjointement au programme, certains cours communs, certaines études axées sur la recherche participative, etc. Les écoles devraient faire en sorte que les cours de recherche ne se limitent pas à la simple présentation de la méthodologie comme la « réalisation de la recherche sociale "; ces cours devraient aussi englober certaines données sur l'utilisation, sur la conversion, sur la création et sur la recherche de développement technique notamment.

En outre, les cours de méthodologie de la recherche usuels devraient se centrer davantage sur les problèmes de la recherche appliquée sur le terrain, sur le travail avec les praticiens à l'intérieur des agences dans le cadre du processus de recherche, sur la réalisation d'études effectuées conjointement par des chercheurs de diverses disciplines, etc. Beaucoup a déjà été accompli en ce sens, mais beaucoup reste à faire. Selon nous, de plus, les écoles devraient participer à la création d'institutions nouvelles ou hybrides : services et centres axés sur l'utilisation de la recherche et sur la R-D. Les écoles de service social pourraient aussi parrainer certains laboratoires de R-D et les écoles pourraient prendre l'initiative d'aider à l'établissement sur le campus de départements d'ingénierie sociale autonomes.

Le rôle de la NASW semble lui aussi très clair : elle pourrait sûrement s'orienter vers une recherche plus respectée et plus reconnue par les membres de la profession. Certaines étapes comme la publication d'une nouvelle revue ont été amorcées dans la bonne voie. Cependant, le scepticisme passé des travailleurs sociaux à l'égard de la recherche s'explique bien : celle qu'on a produite a grandement suivi le schéma classique de l'investigation scientifique et n'a pas respecté 
l'esprit de la recherche médicale ou technique. Une telle démarche était empreinte d'un certain degré de prestige et ce, même au détriment de l'efficacité sur le plan des problèmes traités par les professionnels en service social. La profession peut jouer un rôle prépondérant pour accroitre la recherche tout en insistant concurremment sur son applicabilité face aux problèmes posés par l'intervention et par les programmes. Les chercheurs professionnels devraient obligatoirement y axer leurs travaux sur les programmes professionnels et sur les préoccupations de l'intervention elle-même, de façon claire et directe. Nous ne préconisons nullement le délaissement ou l'affaiblissement de la recherche fondamentale, qui devrait toujours être primordiale surtout pour les spécialistes en sciences sociales. Les professions de services d'aide individualisée pourront toutefois servir à faire contrepoids au sens où nous l'avons expliqué ici. Recherche fondamentale et recherche appliquée jouent toutes deux des rôles de premier plan et doivent être appréciées. La recherche fondamentale a dominé le monde universitaire dans le domaine social : il faut maintenant créer un meilleur équilibre par la mise sur pied d'activités plus nombreuses et par la création d'une méthodologie améliorée dans le domaine appliqué.

La NASW peut favoriser l'amélioration de la qualité de la recherche et accroître son utilité par une multitude d'activités : publications sur le sujet, établissement de comités de traitement d'un problème donné, démarches en vue de créer certains des rôles, certains des mécanismes et certains des rouages institutionnels nouveaux que nous venons d'esquisser et mise en œuvre d'internats et de programmes de bourses d'études pour les jeunes professionnels désireux d'apprendre à remplir de telles fonctions. Peut-être devrait-elle aussi parrainer un congrès de perfectionnement analogue au présent congrès sur l'utilisation de la recherche dans l'enseignement du service social.

Voilà pour les points de départ. Les personnes plus au fait du fonctionnement de cet organisme pourront grandement élargir cette liste, nous n'en doutons point. Quel que soit le chemin que nous empruntions, il nous faut reconnaître que nous devons intégrer certaines fonctions d'ingénierie sociale à nos activités professionnelles, si nous voulons réussir à combler l'écart entre la recherche et les activités d'intervention.

Sceptiques et humanistes pourront être troublés par notre emploi de l'expression «ingénierie sociale». Pour nombre de spécialistes en sciences sociales, ce concept est entaché d'émotivité et donne 
l'impression qu'une méthode froide et technocratique est utilisée dans des domaines d'intérêt social. Qu'il soit bien clair que ce n'est pas là ce que nous voulons signifier ni ce que nous visons. Dans la réalisation de notre travail en R-D sociale au cours des dix dernières années, notre position a été ouverte et tranchée, et nous l'avons exposée dans la première publication officielle du Projet d'intervention communautaire :

\begin{abstract}
Nous avons abordé notre sujet avec certaines conceptions - il faut lire préjugés - qu'il faudrait peut-être expliquer au départ [...] Nous prônons l'élimination des injustices sur le plan social et des iniquités sur le plan économique. Cela suppose donc l'exécution d'un certain travail en vue d'arriver à une organisation sociale où les concepts du bien commun ou du destin collectif de la population s'harmoniseront davantage avec un certain souci avoué "d'individualisme ». En d'autres termes, le bien-être de la collectivité au sens large a plus d'importance sur l'échelle des valeurs humaines que le profil individuel (Rothman, 1974).
\end{abstract}

Nous utilisons l'expression ingénierie sociale de façon délibérée, précise et cohérente, non pas au sens global où on l'emploie souvent. Une telle acception est véhiculée dans la définition même du terme ingénierie donnée par un dictionnaire général :

l'art et la science de la mise en application des connaissances scientifiques, comme c'est le cas au niveau de la conception, de la construction et de l'exploitation de routes, de ponts [...] de réseaux de communication, etc. (Standard College Dictionary, 1966).

Pareille définition met en valeur l'idée de convertir la connaissance scientifique fondamentale à des fins utiles. Selon nous, l'ingénierie sociale est identique au génie physique à cet égard et, en paraphrasant ce qui précède, nous la considérons comme : l'art et la science de la mise en application des connaissances en sciences sociales, comme c'est le cas au point de vue de la conception, de la construction et de l'exploitation de programmes, de techniques et de structures au niveau des agences, etc.

Il est bien évident que l'emploi de cette expression n'est pas sans danger. Dans le passé, on lui a donné des acceptions très vagues et très diverses, et cela peut contribuer à fausser le sens concret et restreint que nous lui donnons ici. 
Au sens où nous la concevons, l'ingénierie sociale vise la solution de problèmes sociaux et le renforcement des membres plus faibles et moins favorisés dans la société. Nous espérons que la R-D sociale contribuera tout particulièrement à promouvoir une distribution de services plus efficace et plus humaine aux membres de la collectivité. Elle crée des outils viables pouvant aider les personnes en ce sens, directement ou indirectement.

Parallèlement, il est vrai que toute technologie nouvelle (énergie nucléaire, vols aérospatiaux, nouveaux médicaments) est intrinsèquement neutre et peut servir à de multiples fins : elle peut avilir l'homme ou le bonifier, le détruire ou l'ennoblir; la technologie sociale ne diffère nullement de la technologie physique à cet égard. Les scientifiques ou les ingénieurs qui inventent une nouvelle technologie n'ont aucun pouvoir sur sa destination ultime. Ils ne peuvent qu'exprimer leurs mobiles et leurs espoirs quant à ses conséquences possibles pour I'humanité. La transmission d'une philosophie donnée pourra peutêtre donner un certain sens à la mise en application de la technologie elle-même.

Pour certains, l'ingénierie dépasse la simple neutralité mécaniste et peut même aller jusqu'au négativisme marqué. En effet, les dictionnaires donnent au terme cette acception généralement reconnue : " Mener à bien ou manier en combinant : machiner un plan [...] manœuvrer » (Standard College Dictionary, 1966).

Le risque d'employer les techniques de R-D avec un tel mépris n'est pas inexistant, mais une telle chose n'est pas inéluctable; dans le cadre de notre propre programme, nous avons pu l'éviter pendant nombre d'années. En effet, nous avons élaboré certaines méthodes de R-D sociale suivant l'hypothèse qu'elles pouvaient aider les professionnels des services sociaux à obtenir, au niveau de la société, des résultats plus bénéfiques qu'ils n'avaient réussi à en obtenir avec les méthodes existantes par l'application planifiée et rigoureuse des connaissances issues de la recherche en sciences sociales.

Comme le présent exposé l'a démontré, il faut tenir compte d'une multiplicité d'éléments sur le plan des concepts, des techniques et des valeurs pour mieux utiliser les connaissances en sciences sociales dans le traitement des problèmes urgents auxquels se heurtent les professionnels du service social. Nous avons laissé entendre que 
pour les explorer et pour progresser dans cette question complexe il fallait transposer, de façon prudente et sélective, la méthodologie mise au point dans le cadre des sciences physiques et de l'industrie, grâce à de longues années d'étude et d'expérimentation en vue de relier les connaissances issues de la recherche aux problèmes appliqués. Pour se légitimer, les professions de services d'aide individualisée en sont maintenant arrivées au stade où elles peuvent cesser de rivaliser avec les sciences sociales fondamentales et de les copier. Les sciences sociales ont atteint une plus grande maturité, elles ont reçu une reconnaissance accrue du public et nous, chercheurs professionnels, avons prouvé notre compétence dans la maîtrise de leurs techniques. Le prochain obstacle à surmonter est l'application rigoureuse des connaissances et des méthodes en sciences sociales : il faut faire le lien entre la méthode de l'ingénierie et celle de la recherche médicale. Un nouvel horizon s'ouvre à nous et nous sommes exceptionnellement bien outillés pour commencer son exploration; c'est à nous qu'il incombe que ce domaine puisse acquérir une certaine crédibilité. Pour les chercheurs en sciences sociales, un signe de maturité grandissante pourrait bien être la reconnaissance de l'importance de cette tâche et un engagement à l'entreprendre.

Jack ROTHMAN

Traduction de René AUCLAIR

Professeur titulaire École de service social Université Laval 


\section{Références bibliographiques}

AMES, Edward (1951). « Communications : Research, Invention, Development and Innovation ", American Economic Review, vol. 51, p. 370-381.

ANSOFF, H.I (1961). " Evaluation of Applied Research in a Business Firm ", dans James R. Bright (dir.), Technological Planning on the Corporate Level, Cambridge, Mass., Harvard University, Graduate School of Business Administration, p. 210-211.

BOYAN, Norman J. (1968). " Problems and Issues of Knowledge Production and Utilization ", dans Terry L. Eiddell et Joanne M. Kitchel (dir.), Knowledge Production and Utilization, Columbus, Ohio, University Council for Educational Administration et Center for the Advanced Study of Educational Administration, Université de l'Oregon, p. 17.

BRooks, Harvey (1965). "Scientific Concepts and Cultural Change ", Daedalus, vol. 94, p. 126.

CARTER, Launor F. (1968). "Knowledge Production and Utilization in Contemporary Organizations ", dans Terry L. Eiddell et Joanne M. Kitchel (dir.)., Knowledge Production and Utilization, Columbus, Ohio, University Council for Educational Administration et Center for the Advanced Study of Educational Administration, Université de l'Oregon, p. 17.

CLARK, David L. (1962). " The Function of the U.S. Office of Education and State Departments of Education in the Dissemination and Implementation of Educational Research », dans K. Goldhammer et S. Elam (dir.), Dissemination and Implementation, Bloomington, Ind., Phi Delta Kappa, p. 105-127.

CRISSWELL, Joan H. (1969). " Research Utilization in Poverty Situations ", Rehabilitation Record, mars-avril, p. 7-11.

CROWE, R.E. et R.S. MAdANCY (1974). The U.S. Environmental Agency's Experience in Technology Transfer, Washington, D.C., Office of Research and Development, U.S. Environmental Protection Agency.

Department of Guidance and Psychological Services (1968). Communication, dissemination and utilization of research information in rehabilitation counseling. Travaux rédigés lors d'une conférence régionale parrainée par le Department of Guidance and Psychological Services, Springfield College. Publication préparée en collaboration avec l'administration des Services de réadaptation, C.R. Cooper et B. Archambault (dir.), Springfield, Mass.

EASH, Maurice J. (1968). “ Bringing Research Findings Into Classroom Practice ", Elementary School Journal, vol. 8. 
ETZIONI, Amitai (1976). "An Earth-NASA : The Agency for Domestic Policy Development ", Human Behavior, vol. 5, décembre, p. 11.

FaR West laboratory for Educational RESEARCH AND DeVElopment (1973). A Training in Educational Development, Dissemination, and Evaluation, Berkeley, CA, FWLERD, mars.

FREY, Donald N. et J.E. GoLDMAN (1967). « Applied Science and Manufacturing Technology », dans Applied Science-Technological Progress, Washington, D.C., National Academy of Sciences, juin.

GLASER, Edward M. et Thomas E. BACKER (1975). " Evaluating the Research Utilization Specialist ", Rehabilitation Counseling Bulletin, vol. 19, décembre, p. 388.

GoRDON, Gerald et Sue Marquis (1966). "Freedom, Visibility of Consequences and Scientific Innovation ", American Journal of Sociology, vol. 72, septembre.

GuBA, Egon G. “ Development, Diffusion and Evaluation », dans Terry L. Eiddell et Joanne M. Kitchel (dir.)., Knowledge Production and Utilization, Columbus, Ohio, University Council for Educational Administration et Center for the Advanced Study of Educational Administration, Université de l'Oregon, p. 39-40.

HALL, Arthur D. (1962). A Methodology for Systems Engineering, New York, D. Van Nostrand Co.

HAVELOCK, Ronald G. (1973). Planning for Innovation Through Dissemination and Utilization of Knowledge, Ann Arbor, Mich., Institute for Social Research, Center for Research on Utilization of Knowledge.

HoLt, Knut (1975). Product Innovation, Trondheim, Université de Trondheim, The Norwegian Institute of Technology, p. 11-61.

KAPPEL, Frederick R. (1976). "Systems Approach in Science-Based Industry ». Écrit reposant sur une allocution présentée au XIII ${ }^{e}$ Congrès de gestion internationale, tenu à New York le 16 septembre 1976.

KELLY, Mervin (1950). "The Bell Telephone Laboratories - An Example of an Institute of Creative Technology ", Proceedings of the Royal Society, Série A, Mathématiques et sciences physiques, 203, n 1074, p. 287-301.

NATIONAL SCIENCE Foundation (1969a). Federal Funds for Research, Development and Other Scientific Activities, vol. 15, exercices financiers de 1966, 1967 et 1968, Washington, D.C., Imprimerie du gouvernement.

NATIONAL SCIENCE FOUNDATION (1969b). Knowledge Into Action: Improving the Nation's Use of the Social Sciences, Washington, D.C., Imprimerie du gouvernement. 
PRICE, James L. (1964). « Use of New Knowledge in Organizations », Human Organization, vol. 23, automne.

RESEARCH UTILIZATION INVENTORY : A Survey of Current Research (19751976). New York, Research Utilization Unit, Community Council of Greater New York City.

ROBERTS, Edward B. (1967). “ Facts and Folklore in Research and Development Management ", Industrial Management Review, vol. 8, p. 5.

RotHMAN, Jack (1977). Research and Development in the Human ServicesToward a Systematic Methodology of Applied Science, Ann Arbor, Mich., Community Intervention Project, Université du Michigan.

SCHUTZ, Richard E. (1979). «The Nature of Educational Development », Journal of Research and Development in Education, vol. 3, p. 39.

Standard College Dictionary (1966). New York, Harcourt, Brace and World.

WOLF, Marta (1976). « Concept of Information Services - Old and New », Contact, vol. 2, printemps-été. 